\title{
NEW RESULTS FROM BHABHA SCATTERING AT 29 GeV
}

M. DERRICK, K.K. GAN, P. KOOIJMAN, J.S. LOOS, B. MUSGRAVE, L.E. PRICE, J. SCHLERETH, K. SUGANO, J.M. WEISS ${ }^{1}$, D.E. WOOD ${ }^{2}$

Argonne National Laboratory, Argonne, IL 60439, USA

D. BLOCKUS, B. BRABSON, S.W. GRAY ${ }^{3}$, C. JUNG, H. NEAL, H. OGREN, D.R. RUST, M. VALDATA-NAPPI ${ }^{4}$

Indiana University, Bloomington, IN 47405, USA

C. AKERLOF, G. BONVICINI, J. CHAPMAN, D. ERREDE, N. HARNEW ${ }^{5}$, P. KESTEN ${ }^{6}$, D.I. MEYER, D. NITZ, A.A. SEIDL ${ }^{2}$, R. THUN, T. TRINKO ${ }^{2}$, M. WILLUTZKY

University of Michigan, Ann Arbor, MI 48109, USA

S. ABACHI, P. BARINGER, I. BELTRAMI ${ }^{7}$, B.G. BYLSMA, R. DEBONTE, D. KOLTICK, F.J. LOEFFLER, E.H. LOW, R.L. McILWAIN, D.H. MILLER, C.R. NG, L.K. RANGAN, E.I. SHIBATA

Purdue University, West Lafayette, IN 47907, USA

and

B. CORK

Lawrence Berkeley Laboratory, Berkeley, CA 94720, USA

Received 31 October 1985

Results are reported on a high statistics study of Bhabha scattering at $29 \mathrm{GeV}$ in the polar angle region, $|\cos \theta|<0.55$. The data are consistent with the standard model, and measure vector and axial-vector coupling constants of $g_{\mathrm{v}}^{2}=0.03 \pm 0.09$ and $g_{a}^{2}=0.46 \pm 0.14$. Limits on the QED-cutoff parameters are $\Lambda_{+}>154 \mathrm{GeV}$ and $\Lambda_{-}>220 \mathrm{GeV}$. Lower limits on scale parameters of composite models are in the range $0.9-2.8 \mathrm{TeV}$. The partial width of a hypothetical spin-zero boson decaying to $\mathrm{e}^{+} \mathrm{e}^{--}$has an upper limit which varies from 6 to $57 \mathrm{MeV}$ corresponding to a boson mass in the range $45-80 \mathrm{GeV} / c^{2}$.

1 Present address: SRI International, Menlo Park, CA 94025, USA.

2 Present address: Lockheed Missiles and Space Co., Sunnyvale, CA 94086, USA.

${ }^{3}$ Present address: Laboratory for Nuclear Studies, Cornell University, Ithaca, NY 14853, USA.

4 Present address: INFN, I-56010 Pisa, Italy.

5 Present address: CERN, CH-1211 Geneva 23, Switzerland.

6 Present address: Brandeis University, Waltham, MA 02254, USA.

${ }^{7}$ Present address: ETH, CH-8093 Zurich, Switzerland.
Bhabha scattering, $\mathrm{e}^{+} \mathrm{e}^{-} \rightarrow \mathrm{e}^{+} \mathrm{e}^{-}$, at high energies has been the subject of much recent experimental work $[1-7]$. This fundamental reaction has been used in testing for possible deviations from the standard model of electroweak interactions (GSW) [8], in setting limits on QED-cutoff parameters [9] and composite model scale parameters [10], and in searching for evidence of spin-zero bosons that couple to $\mathrm{e}^{+} \mathrm{e}^{-}[11,12]$. 
In this paper, results are presented on Bhabha scattering at $29 \mathrm{GeV}$ in the central region of the polar scattering angle, $|\cos \theta|<0.55$. There are 84423 observed events in the present sample acquired with the high resolution spectrome ter (HRS) at the PEP $\mathrm{e}^{+} \mathrm{e}^{-}$ storage ring. Discussions of the detector, experimental trigger, event selections, and corrections for the Bhabha events and a companion reaction, $\mathrm{e}^{+} \mathrm{e}^{-} \rightarrow \gamma \gamma$, are given in the accompanying paper [13]. The $\gamma$ pair data, which are independent of electroweak effects, provide a measurement of the integrated luminosity that has been used in the present study to constrain the Bhabha normalization.

The lowest-order Bhabha differential cross section is given by

$$
\begin{aligned}
& \left(\mathrm{d} \sigma^{0} / \mathrm{d} \Omega\right)=\left(\alpha^{2} / 2 s\right)\left[\left|A_{1}\right|^{2}(s / t)^{2}+\left|A_{2}\right|^{2}(t / s)^{2}\right. \\
& \left.\quad+\frac{1}{2}\left(\left|A_{3}\right|^{2}+\left|A_{4}\right|^{2}\right)(1+t / s)^{2}\right],
\end{aligned}
$$

where $s=E_{\mathrm{CM}}^{2}$ and $t=-s(1-\cos \theta) / 2$. For energies well below the mass of the $Z^{0}$ boson, the electroweak amplitudes are:

$A_{1}=1+\left(g_{\mathrm{v}}^{2}-g_{\mathrm{a}}^{2}\right) \chi(t)$,

$A_{2}=1+\left(g_{\mathrm{v}}^{2}-g_{\mathrm{a}}^{2}\right) \chi(s)$,

$A_{3}=1+s / t+\left(g_{\mathrm{v}}-g_{\mathrm{a}}\right)^{2}[\chi(s)+(s / t) \chi(t)]$,

$A_{4}=1+s / t+\left(g_{\mathrm{v}}+g_{\mathrm{a}}\right)^{2}[\chi(s)+(s / t) \chi(t)]$,

where

$\chi\left(q^{2}\right)=\left(G_{\mathrm{F}} / \pi \alpha \sqrt{8}\right) q^{2} M_{\mathrm{Z}}^{2} /\left(q^{2}-M_{\mathrm{Z}}^{2}\right)$.

$G_{\mathrm{F}}$ is the Fermi coupling constant, $\alpha$ is the finestructure constant, $M_{\mathrm{Z}}$ the mass of the $\mathrm{Z}^{0}, g_{\mathrm{V}}$ the vector coupling, and $g_{\mathrm{a}}$ the axial-vector coupling. Eq. (1) is applicable for any small modification of the amplitudes. In table 1 the modifications of the Bhabha amplitudes tested in this paper are shown in terms of small additions, $\Delta A$, to the amplitudes of eq. (2).

In order to compare theory to experiment, corrections must be made for radiative effects, experimental resolutions, and acceptance. This is done in two steps: (1) the pure QED cross section to order $\alpha^{3}$ (with $g_{\mathrm{a}}=g_{\mathrm{v}}=0$ ) is calculated via the Berends-Kleiss [14] Monte Carlo program which generates simulated events, including the $\mathrm{e}^{+} \mathrm{e}^{-\gamma}$ final state, and (2) experimental resolution smearing and Bhabha event selec-
Table 1

Modifications of the standard model amplitudes for Bhabha scattering that are tested in this paper.

\begin{tabular}{llll}
\hline Amplitude & $\begin{array}{l}\text { QED- } \\
\text { cutoffa) }\end{array}$ & $\begin{array}{l}\text { Composite } \\
\text { model b) }\end{array}$ & $\begin{array}{l}\text { Spin-zero } \\
\text { bosons c) }\end{array}$ \\
\hline$\Delta A_{1}$ & $\pm t / \Lambda_{ \pm}^{2}$ & $\pm \eta_{\mathrm{RL}} t / \alpha^{2} K_{ \pm}^{2}$ & $\epsilon \Gamma_{\mathrm{ee}} t / \alpha M \mathrm{X}^{\left(s-M_{\mathrm{X}}^{2}\right)}$ \\
$\Delta A_{2}$ & $\pm s / \Lambda_{ \pm}^{2}$ & $\pm \eta_{\mathrm{RL}} s / \alpha^{2} K_{ \pm}^{2}$ & $\epsilon \Gamma_{\mathrm{ee}} s / \alpha M \mathrm{X}^{\left(t-M_{\mathrm{X}}^{2}\right)}$ \\
$\Delta A_{3}$ & $\pm 2 s / \Lambda_{ \pm}^{2}$ & $\pm 2 \eta_{\mathrm{RR}} s / \alpha^{2} K_{ \pm}^{2}$ & 0 \\
$\Delta A_{4}$ & $\pm 2 s / \Lambda_{ \pm}^{2}$ & $\pm 2 \eta_{\mathrm{LL}} s / \alpha^{2} K_{ \pm}^{2}$ & 0
\end{tabular}

a) Ref. [9]. b) Ref. [10]. c) Ref. [11].

tions [13] are imposed on the simulated QED events to yield $(\mathrm{d} \sigma / \mathrm{d} \Omega)_{\mathrm{QED}}$. Test differential cross sections, $(\mathrm{d} \sigma / \mathrm{d} \Omega)_{\text {test }}$, corresponding to modified Bhabha scattering amplitudes, are then constructed from the appropriate ratio of analytic second-order cross sections:

$$
(\mathrm{d} \sigma / \mathrm{d} \Omega)_{\text {test }}=(\mathrm{d} \sigma / \mathrm{d} \Omega)_{\mathrm{QED}}
$$

$$
\cdot\left[\left(\mathrm{d} \sigma^{0} / \mathrm{d} \Omega\right)_{\mathrm{test}} /\left(\mathrm{d} \sigma^{0} / \mathrm{d} \Omega\right)_{\mathrm{QED}}\right] \text {. }
$$

Test of the GSW model. First a check is made to see wheter Bhabha scattering is well represented by the standard model. The general expression for the vector coupling is

$g_{\mathrm{v}}^{2}=\left(-\frac{1}{2}+2 \sin ^{2} \theta_{\mathrm{w}}\right)^{2}+4 C$.

In the GSW model, $g_{\mathrm{a}}^{2}=0.25$ and $C=0$. The ratio, $(\mathrm{d} \sigma / \mathrm{d} \Omega)_{\text {expt }} /(\mathrm{d} \sigma / \mathrm{d} \Omega)_{\mathrm{G} \mathrm{SW}}$, is shown in fig. 1a, where the current best estimates $[15,16]$ of the weak parameters $\left(M_{Z}=93 \mathrm{GeV} / c^{2}, \sin ^{2} \theta_{\mathrm{w}}=0.217\right)$ are used. Normalizing the data to the GSW model prediction gives a good fit with $\chi^{2} / n_{\mathrm{D}}=24.1 / 21$, and measures an integrated luminosity of $164.4 \mathrm{pb}^{-1}$ in good agreement with the luminosity found from the $\gamma$ pairs, $L_{0}=165.6 \pm 2.0 \mathrm{pb}^{-1}$ [13].

Next a simultaneous fit is done for $g_{\mathrm{a}}^{2}$ and $g_{\mathrm{v}}^{2}$, fixing the $Z^{0}$ mass at $93 \mathrm{GeV} / c^{2}$. In this fit, and in all other fits done in this paper, the integrated luminosity, $L$, is allowed to vary within the luminosity constraints provided by the $\gamma$-pair events, so that the $\chi^{2}$ function has the form: 


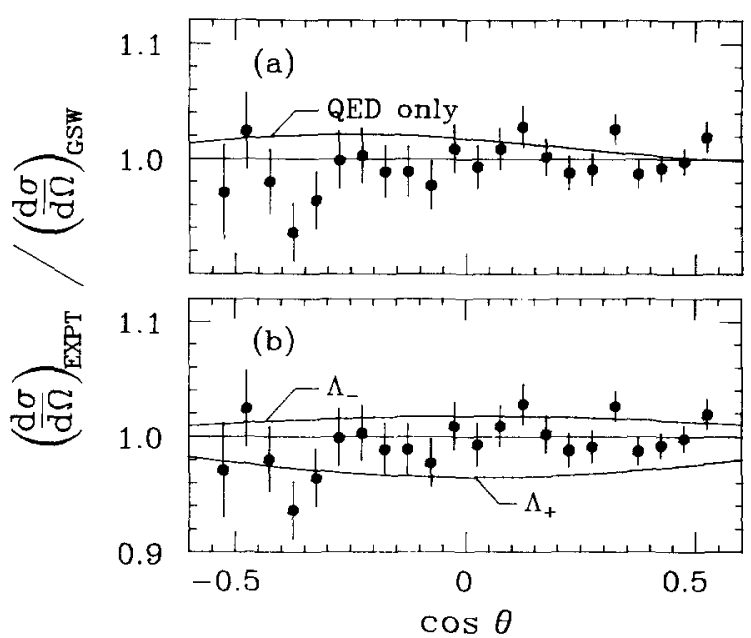

Fig. 1. (a) Ratio of the experimental Bhabha differential cross section to the expected GSW differential cross section at $29 \mathrm{GeV}$. The pure QED result (with $g_{\mathrm{a}}^{2}=g_{\mathrm{v}}^{2}=0$ ) is shown for comparison. (b) The same data are compared to the $95 \%$ confidence lower bounds on the QED-cutoff parameters, $\Lambda_{+}$and $\Lambda_{-}$.

$$
\begin{aligned}
\chi^{2} & =\left[\left(L-L_{0}\right) / \Delta L_{0}\right]^{2} \\
& +\sum_{i}\left(\frac{(\mathrm{d} \sigma / \mathrm{d} \Omega)_{\operatorname{expt}}-\left(L / L_{0}\right)(\mathrm{d} \sigma / \mathrm{d} \Omega)_{\mathrm{test}}}{\delta(\mathrm{d} \sigma / \mathrm{d} \Omega)_{\operatorname{expt}}}\right)_{i}^{2} .
\end{aligned}
$$

The $68 \%$ and $95 \%$ confidence contours in the $\left(g_{\mathrm{a}}^{2}, g_{\mathrm{v}}^{2}\right)$ plane are shown in fig. 2 , and the best fit has $\chi^{2} / n_{\mathrm{D}}=$ 21.6/19. The resulting values for the weak parame ters are $g_{\mathrm{a}}^{2}=0.46 \pm 0.14$ and $g_{\mathrm{v}}^{2}=0.03 \pm 0.09$, which are consistent with the known values. Setting $C=0$ in (4) gives $95 \%$ confidence limits on $\sin ^{2} \theta_{w}$ of $0.02<$ $\sin ^{2} \theta_{\mathrm{w}}<0.48$. Alternatively, if $\sin ^{2} \theta_{\mathrm{w}}=0.217$, then the $95 \%$ confidence limit on $C$, which may be nonzero in some models [17], is $C<0.05$. The PLUTO collaboration [5] has extracted similar results on electroweak parameters from Bhabha scattering.

Although the Bhabha differential cross section is rather insensitive to the weak parameters, the present experiment does provide positive evidence for the existence of the electroweak contributions to this reaction. When $g_{\mathrm{a}}^{2}$ and $g_{\mathrm{v}}^{2}$ are both set to zero, a relatively poor fit to the data is found with $\chi^{2} / n_{\mathrm{D}}=$ $35.4 / 21$. The curve in fig. 1a shows the pure QED expectation for this case.

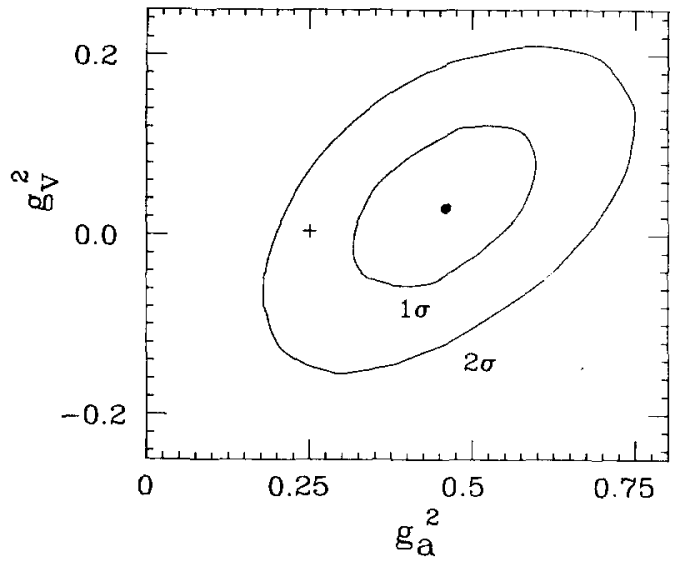

Fig. 2. Plot of the $68 \%$ and $95 \%$ confidence contours in the $\left(g_{\mathrm{a}}^{2}, g_{\mathrm{y}}^{2}\right)$ plane obtained from Bhabha scattering at $29 \mathrm{GeV}$. The dot represents the best fit, whereas the cross shown the GSW model values.

The present experiment, therefore, is consistent with the standard model, and the nominal values $\left(M_{\mathrm{Z}}=93 \mathrm{GeV} / c^{2}, g_{\mathrm{a}}^{2}=0.25\right.$, and $\left.g_{\mathrm{v}}^{2}=0.004\right)$ are assumed in all subsequent fits.

$Q E D$ form factor limits. Limits on the departure of Bhabha scattering from QED are usually parameterized with form factors governed by a cutoff parameter, $\Lambda$ [9], as shown in table 1. A plot of the $68 \%$ and $95 \%$ confidence contours in $E_{\mathrm{cm}} / \Lambda$ versus $L$ is shown in fig. 3. The importance of an accurate, independent measurement of the integrated luminosity is evident in this plot, which shows that the limits on $\Lambda_{ \pm}$are weakened by the correlation between $\Lambda$ and $L$. The $95 \%$ confidence limits found in the present experiment are $\Lambda_{+}>154 \mathrm{GeV}$ and $\Lambda_{-}>220 \mathrm{GeV}$, and the curves in fig. $1 \mathrm{~b}$ correspond to these limits. Note that a QED-cutoff of $\Lambda=200 \mathrm{GeV}$ implies pointlike scattering down to a distance of $10^{-18} \mathrm{~m}$. The present limits on $\Lambda$ are comparable to those obtained in the PETRA experiments $[2,3,5,7]$ at higher energies.

Composite model tests. In the model of Eichten, Lane, and Peskin [10], composite fermions exhibit contact interactions arising from the exchange of constituents. The resulting form-factor modifications to the Bhabha amplitudes are listed in table 1, where $K$ is used to denote the composite model scale param- 


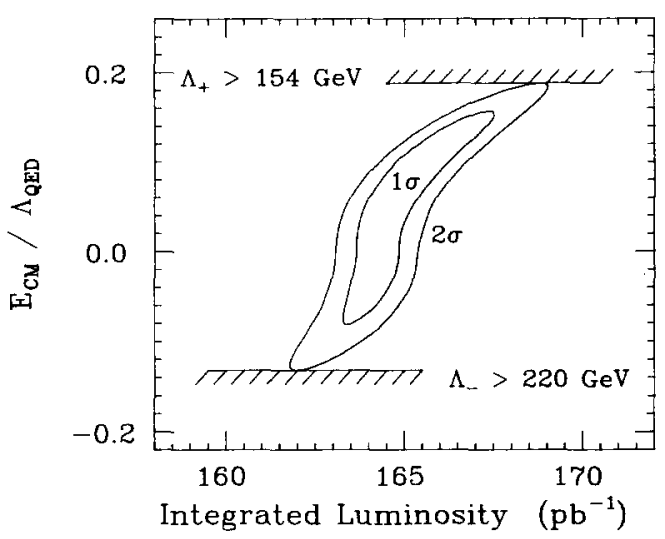

Fig. 3. Plot of the $68 \%$ and $95 \%$ confidence contours for the variable $E_{\mathrm{CM}} / \Lambda$ versus $L$, the integrated luminosity of the sample. $L$ is constrained by an independent measurement from the reaction $\mathrm{e}^{+} \mathrm{e}^{-} \rightarrow \gamma \gamma$. The $\Lambda$ limits for Bhabha scattering quoted in the present paper are shown.

eters. Contributions from the helicity-conserving contact interactions may occur in several different combinations. The following cases are considered by Eichten et al.:

(a) left handed currents (LL): $\eta_{\mathrm{LL}}= \pm 1, \eta_{\mathrm{R} R}=$ $\eta_{\mathrm{RL}}=0$,

(b) right-handed currents (RR): $\eta_{\mathrm{RR}}= \pm 1, \eta_{\mathrm{LL}}=$ $\eta_{\mathrm{RL}}=0$,

(c) vector current (VV): $\eta_{\mathrm{LL}}=\eta_{\mathrm{R} R}=\eta_{\mathrm{RL}}= \pm 1$,

(d) axial-vector currents (AA): $\eta_{\mathrm{LL}}=\eta_{\mathrm{R} R}=$ $-\eta_{\mathrm{RL}}= \pm 1$.

The $95 \%$ confidence lower limits found in this experiment are summarized in table 2 . The $\mathrm{LL}$ and RR cases are not distinguishable in Bhabha scattering.

Table 2

Lower bounds (95\% confidence) for the QED-cutoff parameters $(\Lambda)$ and the composite model scale parameters $(K)$ obtained from Bhabha scattering at $29 \mathrm{GeV}$.

\begin{tabular}{llll}
\hline & \multicolumn{1}{c}{$\Lambda_{+}(\mathrm{GeV}) \Lambda_{-}(\mathrm{GeV}) K_{+}(\mathrm{TeV}) K_{-}(\mathrm{TeV})$} \\
\hline QED-cutoff & 154 & 220 & \\
& & & \\
composite model & & & \\
(LL, RR) & & 1.17 & 0.87 \\
(VV) & 1.80 & 2.58 \\
(AA) & & 2.79 & 1.30 \\
\hline
\end{tabular}

The VV case reduces to the same form as in the QEDcutoff amplitude, with $\Lambda^{2}=\alpha K^{2}$.

The limits given in table 2 are based on an event sample nine times larger than that used in an earlier HRS analysis [1], and so are improved over the earlier work. Similar results have been reported recently by other experiments $[5,7]$.

Spin-zero mesons. The possible existence of neutral, massive spin-zero particles, $X^{0}$, which couple to $\mathrm{e}^{+} \mathrm{e}^{-}$has been proposed recently $[11,12]$. No evidence for such particles has been found in direct $\mathrm{e}^{+} \mathrm{e}^{-}$annihilations up to $46.5 \mathrm{GeV}[2,6,7]$. If such particles exist with masses above this energy, then the Bhabha amplitudes could be altered by the tail of a Breit-Wigner term, as shown in table 1. In the model of Hollik et al., the bosons occur as a scalarpseudoscalar doublet, such that the partial width of $\mathrm{X}^{0} \rightarrow \mathrm{e}^{+} \mathrm{e}^{-}$is given by $\epsilon \Gamma_{\mathrm{ee}}$, where $\epsilon=2$ if the doublet members have the same mass, $M_{\mathrm{X}}$, and $\epsilon=$ 1 if one of the masses is too large to influence the present data.

Upper limits (95\% confidence) on $\epsilon \Gamma_{\mathrm{ee}}$ as a function of $M_{\mathrm{X}}$, shown in fig. 4 , range from $6 \mathrm{MeV}$ at $M_{\mathrm{X}}=45 \mathrm{GeV} / c^{2}$ to $57 \mathrm{MeV}$ at $M_{\mathrm{X}}=80 \mathrm{GeV} / c^{2}$. Other experiments [2,5-7] report similar limits on the partial width.

In summary, the present results on Bhabha scattering at $29 \mathrm{GeV}$ are consistent with the standard model of electroweak interactions, but are not well represented by pure QED (i.e., with $g_{\mathrm{a}}^{2}=g_{\mathrm{v}}^{2}=0$ ). The data are also used to set limits on possible deviations

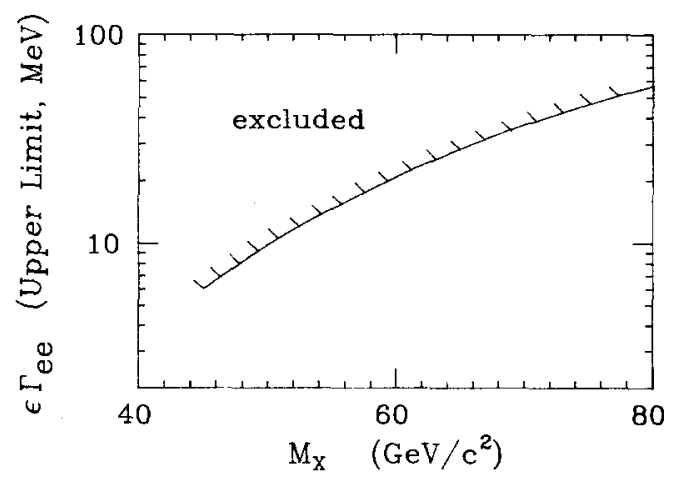

Fig. 4. Upper limit (95\% confidence) for the partial width of a hypothetical spin-zero boson of mass, $M_{\mathrm{X}}$, decaying to $e^{+} e^{-}$. 
from the standard model, which are expressed in terms of QED-cutoff parameters, composite models, and hypothetical spin-zero particles at high mass. These limits are similar to those reported by the PETRA experiments $[2,3,5-7]$ at energies above 34 $\mathrm{GeV}$, and therefore provide a valuable confirmation of the PETRA results. It should be noted that such deviations are approximately proportional to $s$, and that the present experiment has achieved the same sensitivity as the PETRA experiments by having more events at large polar angles and by having better control of systematic uncertainties.

This work was supported in part by the US Department of Energy, under Contracts W-31-109. ENG-38, DE-AC02-76ER01112, DE-AC0376SF00098, DE-AC02-76ER01428, and DE-AC0284ER40125. The outstanding support of the technical staffs at SLAC and the collaborating institutions have made this experiment possible. We thank G. Bodwin for an interesting and informative discussion.

\section{References}

[1] HRS Collab., D. Bender et al., Phys. Rev. D30 (1984) 515.

[2] MARK J. Collab., B. Adeva et al., Phys. Lett. 152B (1985) 439; Phys. Rev. Lett. 53 (1984) 134.

[3] JADE Collab., W. Bartel et al., Z. Phys. C19 (1983) 197.
[4] MARK II Collab., M.E. Levi et al., Phys. Rev. Lett. 51 (1983) 1941.

[5] PLUTO Collab., Ch. Berger et al., Z. Phys. C27 (1985) 341.

[6] CELLO Collab., H.-J. Behrend et al., Phys. Lett. 140B (1984) 130;Z. Phys. C16 (1983) 301.

[7] TASSO Collab., M. Althoff et al., Phys. Lett. 154B (1985) 236;Z. Phys. C22 (1984) 13.

[8] S.L. Glashow, Nucl. Phys. 22 (1961) 579; A. Salam and J.C. Ward, Phys. Lett. 13 (1964) 168; S. Weinberg, Phys. Rev. Lett. 19 (1967) 1264; Phys. Rev. D5 (1972) 1412.

[9] S.D. Drell, Ann. Phys. 4 (1958) 75.

[10] E.J. Eichten, K.D. Lane and M.E. Peskin, Phys. Rev. Lett. 50 (1983) 811.

[11] W. Hollik, F. Schrempp and B. Schrempp, Phys. Lett. 140B (1984) 424.

[12] R.D. Peccei, Phys. Lett. 136B (1984) 121; F.M. Renard, Phys. Lett. 139B (1984) 449; F.W. Bopp et al., Siegen University preprint SI 84-3 (1984), unpublished.

[13] HRS Collab., M. Derrick et al., Phys. Lett. 166B (1986) 468.

[14] F.A. Berends and R. Kleiss, Nucl. Phys. B228 (1983) 537.

[15] WJ. Marciano and S. Sirlin, Phys. Rev. D29 (1984) 945.

[16] UA1 Collab., C. Arnison et al., Phys. Lett. 147B (1984) 241; UA2 Collab., M. Bagnaia et al., Phys. Lett. 129B (1983) 130.

[17] P.Q. Hung and J.J. Sakurai, Nucl. Phys. B143 (1978) 81; J.D. Bjorken, Phys. Rev. D19 (1979) 335;

E.H. deGroot et al., Phys. Lett. 95B (1980) 128; V. Barger et al., Phys. Rev. D22 (1980) 727. 